\title{
What Is the Optimal Timing of Bowel Preparation for Video Capsule Endoscopy?
}

\author{
Hyun Joo Song ${ }^{1}$ and Ki-Nam Shim ${ }^{2}$ \\ ${ }^{1}$ Department of Internal Medicine, Jeju National University School of Medicine, Jeju, ${ }^{2}$ Department of Internal Medicine, Ewha Womans University \\ School of Medicine, Seoul, Korea
}

See "A Single-Center Randomized Controlled Trial Evaluating Timing of Preparation for Capsule Enteroscopy" by Katherine R. Black, Wiley Truss, Cynthia I. Joiner, Shajan Peter and Frederick H. Weber Jr, on page 234-238.

Bowel preparation prior to video capsule endoscopy (VCE) is as essential as bowel preparation prior to colonoscopy, since several factors, such as food material, air bubbles in the small bowel and delayed gastric and small bowel transit time (SBTT) influence the small bowel visualization quality (SBVQ), diagnostic yield (DY), and completion rate (CR). The 2009 European Society of Gastrointestinal Endoscopy guidelines advise that purgative bowel preparations enhance the DY of a small bowel examination using VCE and do not affect the VCE CR (category of evidence 2a; grade of recommendation B).

According to the 2013 Korean guidelines from the Korean Gut Image Study Group, ${ }^{2}$ bowel preparation with polyethylene glycol (PEG) solution enhances SBVQ and DY, but has no effect on cecal CR. Bowel preparation with $2 \mathrm{~L}$ of PEG solution is similar to that with $4 \mathrm{~L}$ of PEG in terms of the SBVQ, DY, and CR of VCE. Bowel preparation with fasting or PEG solution combined with simethicone enhances the SBVQ, but does not affect the CR of VCE. Bowel preparation with prokinetics does not enhance the SBVQ, DY, or CR of VCE. A 2 L PEG-based purge, administered the day before the procedure, is the most widely practiced preparation regimen. ${ }^{3}$ To date, there is no consensus regarding the optimal timing of bowel preparation for VCE. ${ }^{4}$

Black et al. ${ }^{5}$ conducted a single-center randomized controlled trial evaluating preparation for VCE. Thirty-four patients were randomized prospectively into two equal groups accord-

Received: April 21, 2015 Accepted: April 27,2015

Correspondence: Ki-Nam Shim

Department of Internal Medicine, Ewha Womans University School of Medicine, 1071 Anyangcheon-ro, Yangcheon-gu, Seoul 158-710, Korea

Tel: +82-2-2650-2632, Fax: +82-2-2655-2076, E-mail: shimkn@ewha.ac.kr

(c) This is an Open Access article distributed under the terms of the Creative Commons Attribution Non-Commercial License (http://creativecommons.org/ licenses/by-nc/3.0) which permits unrestricted non-commercial use, distribution, and reproduction in any medium, provided the original work is properly cited. ing to the timing of intestinal lavage (2 L of PEG). Group A had the intestinal lavage over 2 hours, beginning 14 hours before the scheduled VCE, and group B began ingestion 4 hours prior to the study. The results regarding mucosal abnormalities (77\% vs. $82 \%, p=$ not significant [NS]) and DY ( $47 \%$ vs. $53 \%$, $p=\mathrm{NS}$ ) were similar for both groups. There was no significant association between the quality and timing of small bowel preparation. The day before intestinal lavage was similar to same-day preparation with respect to overall preparation quality, CR, SBTT, frequency of identified mucosal abnormalities, and overall DY.

To the best of our knowledge, this is the first study to consider the timing of bowel preparation for VCE and is the first to assess the optimal 'timing' for initiating intestinal lavage in VCE. As mentioned, timing of purgative administration may be a critical factor for visualization and VCE CRs. Most purgative studies have administered purgatives the day prior to the VCE. ${ }^{6-10}$ A Korean study compared fasting for 12 hours and receiving 2 or $4 \mathrm{~L}$ of PEG 4 hours prior to PEG. It showed that bowel preparation with PEG resulted in a better image quality than fasting alone, ${ }^{11}$ but no significant difference was observed between 2 and $4 \mathrm{~L}$ of PEG.

With respect to bowel preparation for the colonoscopy, the quality of bowel preparation is significantly better when there is a shorter time between bowel preparation and the start of colonoscopy. ${ }^{12}$ A shorter ( $<8$ hours) interval between the end of bowel preparation and the start of colonoscopy yielded better bowel cleansing than a longer ( $>8$ hours) interval. ${ }^{13}$ Since the small intestine is anatomically proximal, the authors hypothesized that a similar lavage timing concept would be advantageous for VCE small intestinal visualization and perhaps transit. The overall evidence base for small bowel VCE is immature and validated scales for quality of cleansing are not yet 
available. ${ }^{4}$ One of the issues for bowel preparation of VCE is that the distal segment of the small intestine should be improved. However, there was no significant difference between the two groups (day-before VCE vs. 4 hours prior to VCE) in this study. ${ }^{5}$

Recently, different kinds of bowel preparation such as PEG, PEG plus ascorbic acid, sodium picosulfate and phosphate (NaP) have become available. ${ }^{14} \mathrm{NaP}$ is not generally recommended for bowel cleansing due to the potential for renal damage and other adverse events. However, the use of $\mathrm{NaP}$ is acceptable in patients for whom PEG or sodium picosulfate is ineffective or not tolerated. ${ }^{4}$ There was no published evidence to support the use of sodium picosulfate; however, it is used in some units. Anecdotal evidence suggests that it is not as effective as PEG. ${ }^{4}$ For each of these agents, a consensus should be reached regarding the optimal timing of bowel preparation.

The main limitation of this study is that the number of patients $(n=34)$ is not sufficient for generalization to actual practice. Therefore, a large, multicenter randomized controlled trial is needed to clarify the optimal timing of bowel preparation for VCE. Guidelines for timing of bowel preparation should be made in the near future.

\section{Conflicts of Interest}

The authors have no financial conflicts of interest.

\section{REFERENCES}

1. Ladas SD, Triantafyllou K, Spada C, et al. European Society of Gastrointestinal Endoscopy (ESGE): recommendations (2009) on clinical use of video capsule endoscopy to investigate small-bowel, esophageal and colonic diseases. Endoscopy 2010;42:220-227.
2. Song HJ, Moon JS, Do JH, et al. Guidelines for bowel preparation before video capsule endoscopy. Clin Endosc 2013;46:147-154.

3. Koulaouzidis A, Rondonotti E, Karargyris A. Small-bowel capsule endoscopy: a ten-point contemporary review. World J Gastroenterol 2013; 19:3726-3746

4. Mathus-Vliegen E, Pellisé M, Heresbach D, et al. Consensus guidelines for the use of bowel preparation prior to colonic diagnostic procedures: colonoscopy and small bowel video capsule endoscopy. Curr Med Res Opin 2013;29:931-945.

5. Black KR, Truss W, Joiner CI, Peter S, Weber FH Jr. A single-center randomized controlled trial evaluating timing of preparation for capsule enteroscopy. Clin Endosc 2015;48:234-238.

6. Spada C, Riccioni ME, Familiari P, et al. Polyethylene glycol plus simethicone in small-bowel preparation for capsule endoscopy. Dig Liver Dis 2010;42:365-370.

7. van Tuyl SA, den Ouden H, Stolk MF, Kuipers EJ. Optimal preparation for video capsule endoscopy: a prospective, randomized, single-blind study. Endoscopy 2007;39:1037-1040.

8. Viazis N, Sgouros S, Papaxoinis K, et al. Bowel preparation increases the diagnostic yield of capsule endoscopy: a prospective, randomized, controlled study. Gastrointest Endosc 2004;60:534-538.

9. Wei W, Ge ZZ, Lu H, Gao YJ, Hu YB, Xiao SD. Purgative bowel cleansing combined with simethicone improves capsule endoscopy imaging. Am J Gastroenterol 2008;103:77-82.

10. Wi JH, Moon JS, Choi MG, et al. Bowel preparation for capsule endoscopy: a prospective randomized multicenter study. Gut Liver 2009;3: 180-185.

11. Park SC, Keum B, Seo YS, et al. Effect of bowel preparation with polyethylene glycol on quality of capsule endoscopy. Dig Dis Sci 2011;56: 1769-1775.

12. Eun CS, Han DS, Hyun YS, et al. The timing of bowel preparation is more important than the timing of colonoscopy in determining the quality of bowel cleansing. Dig Dis Sci 2011;56:539-544.

13. Bryant RV, Schoeman SN, Schoeman MN. Shorter preparation to procedure interval for colonoscopy improves quality of bowel cleansing. Intern Med J 2013;43:162-168.

14. Pons Beltrán V, Carretero C, Gonzalez-Suárez B, Fernandez-Urien I, Muñoz-Navas M. Intestinal preparation prior to capsule endoscopy administration. World J Gastroenterol 2008;14:5773-5775. 